\title{
Resource Scheduling in Dependable Integrated Modular Avionics
}

\author{
Yann-Hang Lee and Daeyoung Kim \\ Real Time Systems Research Laboratory \\ CISE Department, University of Florida \\ \{yhlee, dkim\}@cise.ufl.edu
}

\author{
Mohamed Younis, Jeff Zhou, James McElroy \\ Honeywell International Inc. \\ \{mohamed.younis, jeff.zhou, james.mcelroy\} \\ @honeywell.com
}

\begin{abstract}
In the recent development of avionics systems, Integrated Modular Avionics (IMA) is advocated for next generation architecture that needs integration of mixedcriticality real-time applications. These integrated applications meet their own timing constraints while sharing avionics computer resources. To guarantee timing constraints and dependability of each application, an IMA-based system is equipped with the schemes for spatial and temporal partitioning. We refer the model as SP-RTS (Strongly Partitioned Real-Time System), which deals with processor partitions and communication channels as its basic scheduling entities.

This paper presents a partition and channelscheduling algorithm for the SP-RTS. The basic idea of the algorithm is to use a two-level hierarchical schedule that activates partitions (or channels) following a distance-constraints guaranteed cyclic schedule and then dispatches tasks (or messages) according to a fixed priority schedule. To enhance schedulability, we devised heuristic algorithms for deadline decomposition and channel combining. The simulation results show the schedulability analysis of the two-level scheduling algorithm and the beneficial characteristics of the proposed deadline decomposition and channel combining algorithms.
\end{abstract}

\section{Introduction}

Advances in computer and communication technology have introduced new architectures for avionics systems, which emphasize the integration of applications, dependability, and cost reduction. Away from the traditional federated implementation for avionics systems, the new approach, referred to as Integrated Modular Avionics (IMA) [1], utilizes multiple standardized processor modules in building functional components of avionics systems. It allows the applications to be merged into an integrated system. While permitting resource sharing, the approach employs temporal and spatial partitioning to set up the application boundaries needed to maintain system predictability, real-time response, and dependability $[2,6]$. For the interactions between applications, it adopts a message model that can easily accommodate replicated executions of mission-critical applications.

Under the IMA architecture, each processor can host multiple partitions in which applications can be executed using the assigned resources. Spatial partitioning implies that a partition cannot access other partition's resources, like memory, buffers, and registers. On the other hand, temporal partitioning guarantees a partition's monopoly use of a pre-allocated processing time without any intervention from other partitions. Thus, a partition is the sole owner of its resources, such as memory segments, $\mathrm{I} / \mathrm{O}$ devices, and processor time slots. As a result, the applications running in different partitions cannot interfere with each other. To facilitate communications between applications, each partition can be assigned with one or more communication channels. An application can transmit messages during the slots allocated to its channel and access exclusively the channel buffers. In this sense, the channels are spatial and temporal partitions of communication resource and are dedicated to one message-sending application.

An application running within a partition can be with multiple cooperating tasks. For instance, the Honeywell's Enhanced Ground Proximity Warning System (EGPWS) consists of tasks for map loading, terrain threat detection, alert prioritization, display processing, etc. With the spatial and temporal partitioning, the EGPWS application can be developed separately and then integrated with other applications running in different partitions of an IMA-based system. Its execution cannot be affected by any malfunctions of other applications (presumably developed by other manufactures) via wild writes or task overruns. However, sufficient resources must be allocated to the partition and the channels, so that the EGPWS application can ensure a proper execution and meet its real-time constraints.

One apparent advantage of IMA-based systems with spatial and temporal partitioning is that each application is running in its own environment. Thus, as long as the 
partition environment is not changed, an application's behavior remains constant even if other applications are modified. This leads to a crucial advantage to avionics systems, i.e. when one application is revised, other applications don't need to be re-certified by the FAA. Thus, the integration of applications in a complex system can be upgraded and maintained easily. It is conceivable that such architecture with spatial and temporal partitioning can be useful for integrating general real-time applications, and will be referred to as a strongly partitioned real-time system (SP-RTS) in the paper.

In this paper, we investigate the issues related to the partition and channel scheduling in SP-RTS. To schedule processor execution, we need to determine which partition is active and to select a task from the active partition for execution. According to temporal partitioning, time slots are allocated to partitions. Within each partition, fixed priorities are assigned to tasks based on rate-monotonic or deadline-monotonic algorithms $[14,5]$. A lower priority task can be preempted by higher priority tasks of the same partition. In other words, the scheduling approach is hierarchical that partitions are scheduled following a cyclic schedule and tasks are dispatched according to a fixed priority schedule. We can conjecture a real system where partitions are processes with protected memory spaces and tasks are threads in a process. At process level, a cyclic scheduling is employed, whereas, in thread level, thread priorities are compared. The scheme doesn't need to make a global priority comparison between threads of different processes. Similar hierarchical scheduling is also applied to the communication media where channels are scheduled in a cyclic fashion and have enough bandwidth to guarantee message communication. Within each channel, messages are then ordered according to their priorities for transmission.

Given task execution characteristics, we are to determine the cyclic schedules for partitions and channels under which the computation results can be delivered before or on the task deadlines. The problem differs from the typical cyclic scheduling since, at the partition and channel levels, we don't evaluate the invocations for each individual task or message. Only aggregated task execution and message transmission models are considered. In addition, the scheduling for partitions and channels must be done collectively such that tasks can complete their computation and then send out the results without missing any deadlines.

A different two-level hierarchical scheduling scheme has been proposed by Deng and Liu in [8]. The scheme allows real-time applications to share resources in an open environment. The scheduling structure has an earliestdeadline-first (EDF) scheduling at the operating system level. The second level scheduling within each application can be either time-driven or priority-driven. For acceptance test and admission of a new application, the scheme analyzes the application schedulability at a slow processor. Then, the server size is determined and server deadline of the job at the head of the ready queue is set at run-time. Since the scheme does not rely on fixed allocation of processor time or fine-grain time slicing, it can support various types of applications, such as release time jitters, non-predictable scheduling instances, and stringent timing requirements.

The scheduling approach for avionics applications under the APEX interface of IMA architecture was discussed by Audsley and Wellings [4]. A recurrent solution to analyze task response time in an application domain is derived and the evaluation results show that there is a potential for a large amount of release jitter. However, the paper does not address the issues of constructing cyclic schedules at the operating system level. To remedy the problem, our first step is to establish scheduling requirements for the cyclic schedules such that task schedulability under a given fixed priority schedules within each partition can be ensured. The approach we adopt is similar to the one in [8] of comparing the task execution in SP-RTS environment with that at a dedicated processor. The cyclic schedule then tries to allocate partition execution intervals by "stealing" task inactivity periods. This stealing approach resembles the slack stealer for scheduling soft-aperiodic tasks in fixed priority systems [11]. Once the schedulability requirements are obtained, suitable cyclic schedules can be constructed. Following the partitioning concept of IMA, the operating system level cyclic schedule is flexible to support system upgrade and integration. It is designed in a way that no complete revision of scheduling algorithms is required when the workload or application tasks in one partition are modified.

The rest of the paper is organized as follows. In section 2, we describe the system models that describe tasks, partition servers, messages, and channel servers in SPRTS. Then, we show the overall system scheduling algorithm and its specific components, such as deadline decomposition, task and message schedulability checking, channel combining, and cyclic scheduling for partition servers and channel servers in section 3. Evaluation results are presented in section 4. A conclusion is then given in section 5 .

\section{System Models}

The SP-RTS system model, as shown in Figure 1, includes multiple processors inter-connected by a time division multiplexing communication bus such as ARINC 659 [3]. Each processor has several execution partitions to which applications can be allocated. An application consists of multiple concurrent tasks that can communicate with each other within the application partition. Task execution is subject to deadlines. Each 
task must complete its computation and send out the result messages on time in order to meet its timing constraints. Messages are the only form of communication among applications, regardless of whether their execution partitions are in the same processor or not. For interpartition communication, the bandwidth of the shared communication media is distributed among all applications by assigning channels to a subset of tasks running in a partition. We assume that there are hardware mechanisms to enforce the partition environment and channel usage by each application, and to prevent any unauthorized accesses. Thus, task computation and message transmission are protected in their application domain. The mechanisms could include memory protection controller, slot/channel mapping, and separate channel buffers.

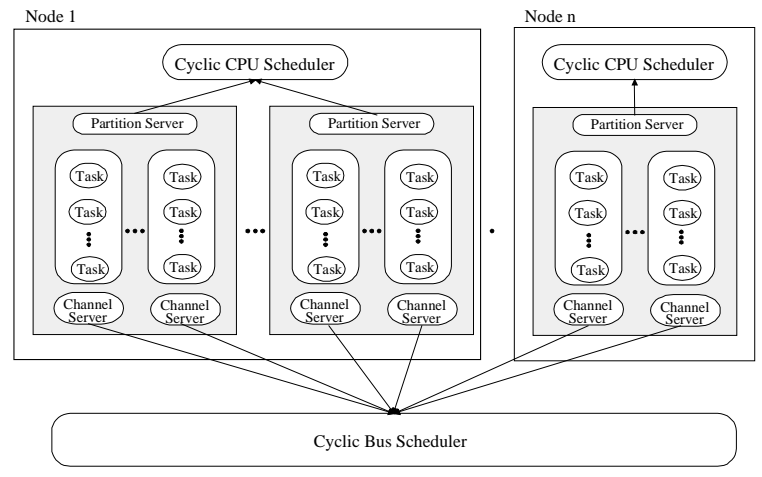

Figure 1. The architecture model for strongly partitioned real-time systems (SP-RTS)

In our task model, we assume that each task arrives periodically and needs to send an output message after its computation. Thus, as illustrated in Figure 2, tasks are specified by several parameters, including invocation period $\left(T_{i}\right)$, worst-case execution time $\left(C_{i}\right)$, deadline $\left(D_{i}\right)$ and message size $\left(M_{i}\right)$. Note that, to model sporadic tasks, we can assign the parameter $T_{i}$ as the minimum inter-

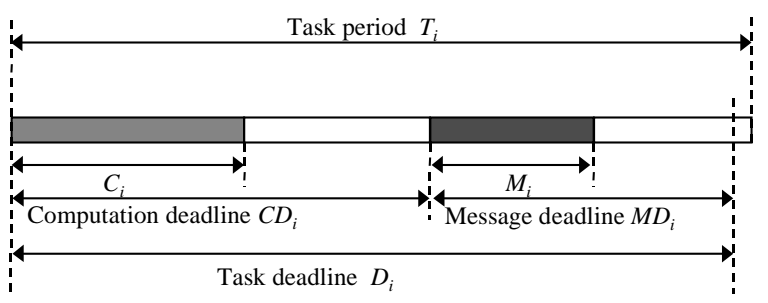

Figure 2. Task model and deadlines

arrival interval between two consecutive invocations.

In order to schedule tasks and messages at processors and communication channels, the task deadline, $D_{i}$, is decomposed into message deadline $\left(M D_{i}\right)$ and computation deadline $\left(C D_{i}\right)$. The assignment of message deadlines influences the bandwidth allocation for the message. For example, when the message size, $M_{i}$, is $1 \mathrm{~K}$ slots, and the message deadline of $10 \mathrm{~ms}$, then the bandwidth requirement is $0.1 \mathrm{M}$ slots per second. In the case of the $1 \mathrm{~ms}$ message deadline, the bandwidth requirement becomes $1 \mathrm{M}$ slots per second. However, a tradeoff must be made since a long message deadline implies a less amount of bandwidth to be allocated, thus the task computation has to be completed immediately.

For each processor in SP-RTS architecture, the scheduling is done in a two-level hierarchy. The first level is within each partition server where the application tasks are running and a higher priority task can preempt any lower priority tasks of the same partition. The second level is a cyclic partition schedule that allocates execution time to partition servers of the processor. In other word, each partition server, $S_{k}$, is scheduled periodically with a fixed period. We denote this period as the partition cycle, $\eta_{k}$. For each partition cycle, the server can execute the tasks in the partition for an interval $\alpha_{k} \eta_{k}$ where $\alpha_{k}$ is less than or equal to 1 and is called partition capacity. For the remaining interval of $\left(1-\alpha_{k}\right) \eta_{k}$, the server is blocked. In Figure 3, an example execution sequence of a partition that consists of three tasks is depicted. During each partition cycle, $\eta_{k}$, the tasks, $\tau_{1}, \tau_{2}$, and $\tau_{3}$, are scheduled to be executed for a period of $\alpha_{k} \eta_{k}$. If there is no active task in the partition, the processor is idle and cannot run any active tasks from other partitions.

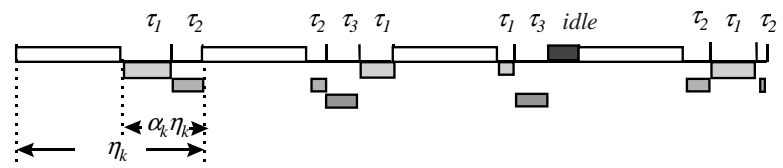

Figure 3. An illustrative task and partition execution sequence

Similarly, a two-level hierarchical scheduling method is applied to the message and channel scheduling. A channel server provides fixed-priority preemptive scheduling for messages. Then, a cyclic schedule assigns a sequence of communication slots to each channel server according to its channel cycle, $\mu_{k}$, and channel capacity, $\beta_{k}$. A channel may send out messages using $\beta_{k} \mu_{k}$ slots during every period of $\mu_{k}$ slots. Note that we use the unit of "slot" to indicate both message length and transmission time, with an assumption that communication bandwidth and slot length are given. For instance, a 64-bit slot in the $30 \mathrm{MHz}$ 2-bit wide ARINC 659 bus [3] is equivalent to $1.0667 \mu \mathrm{s}$, and a message of 1000 bytes will be transmitted in 125 slots. For convenience purposes, we define the conversion factors $S T$ as a slot-to-time ratio based on slot length and bus bandwidth. 


\section{Scheduling Approach}

The objective of our scheduling approach is to find feasible cyclic schedules for partition and channel servers which process tasks and transmit messages according to their fixed priorities within the servers. With proper capacity allocation and frequent invocation at each server, the combined delays of task execution and message transmission are bounded by the task deadlines. In Figure 4, we show the overall approach which first applies a heuristic deadline decomposition to divide the problem into two parts: partition-scheduling and channelscheduling. If either one cannot be done successfully, the approach iterates with a modified deadline assignment. We also assume that the initial task set imposes a processor utilization and a bus utilization less than $100 \%$ and each task's deadline is larger than its execution time plus its message transmission time, i.e., $D_{i} \geq C_{i}+S T * M_{i}$ for task $i$.

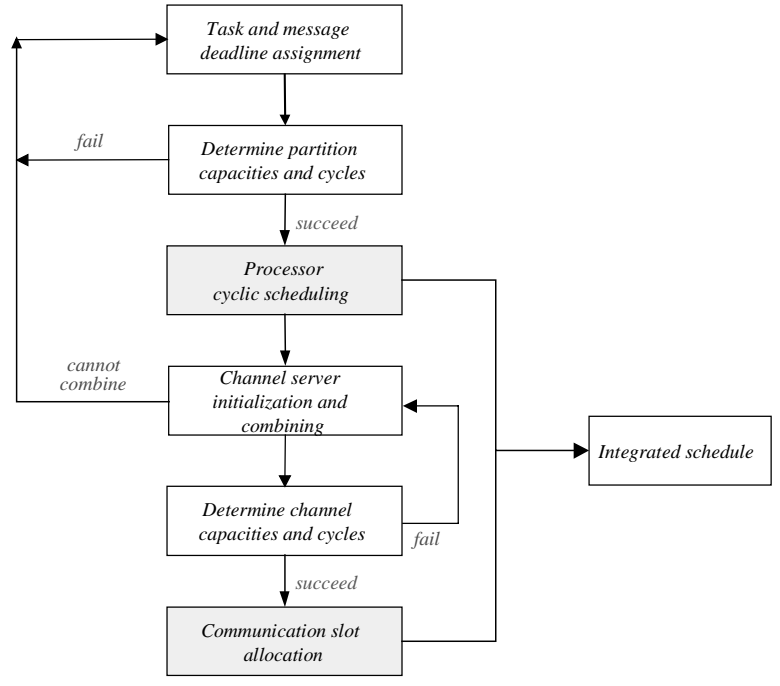

Figure 4. Combined partition and channel scheduling approach

\subsection{Deadline Decomposition}

It is necessary to decompose the original task deadline, $D_{i}$, into computation and message deadline, $C D_{i}$ and $M D_{i}$, for every task, before we can schedule the servers for partition execution and message transmission. A deadline decomposition algorithm is used to assign these deadlines in a heuristic way. If we assign tight message deadlines, messages may not be schedulable. Similarly, if tasks have tight deadlines, processor scheduling can fail. The following equation is used to calculate the message deadline and computation deadline for each task:

$$
\begin{aligned}
& \text { Message Deadline, } M D_{i}=\left(D_{i} \frac{S T * M_{i}}{C_{i}+S T * M_{i}}\right) f_{i} \\
& \text { Computation Deadline, } C D_{i}=D_{i}-M D_{i}
\end{aligned}
$$

where $f_{i}$ is an adjusting factor for each task. The main idea of deadline decomposition is that it allocates the deadlines, $C D_{i}$ and $M D_{i}$, proportionally to their time requirements needed for task execution and message transmission. In addition, the adjusting factor $f_{i}$ is used to calibrate the computation and message deadlines based on the result of previous scheduling attempts and the utilization at processor and communication bus. Since the message and task deadlines must be lower-bounded to the transmission time $\left(S T * M_{i}\right)$ and computation time $\left(C_{i}\right)$, respectively, and upper-bounded to $D_{i}$, we can obtain the lower bound and upper bound of the adjusting factor $f$ as

$$
\frac{1}{D_{i}\left(\frac{1}{C_{i}+S T * M_{i}}\right)} \leq f_{i} \leq \frac{D_{i}-C_{i}}{D_{i}\left(\frac{S T * M_{i}}{C_{i}+S T * M_{i}}\right)}
$$

Since an adjusting factor of 1.0 is a fair distribution and always included in the range of $f_{i}$, we set the initial value of $f_{i}$ to be 1 . The heuristic deadline decomposition, as show in Figure 5, is similar to a binary search algorithm in the attempt of finding the right proportion of task and message deadlines. If we reach the situation that it cannot assign new value for all tasks, we declare the input set of tasks as unschedulable.

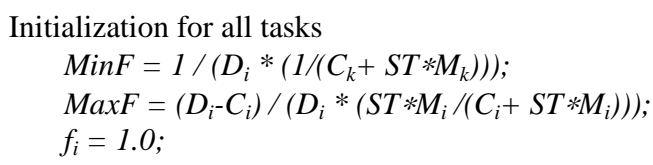

Iterative change of $f_{k}$ when either partition or channel scheduling fails

If (Partition scheduling fails) \{ \} $\operatorname{MaxF}=f_{i} ; f_{i}=\left(\operatorname{MinF}+f_{i}\right) / 2.0 ;$ else if (Channel scheduling fails) \{ \} $\operatorname{MinF}=f_{i} ; f_{i}=\left(\operatorname{Max} F+f_{i}\right) / 2.0 ;$

Figure 5. The deadline decomposition algorithm

\subsection{Partition and Channel Scheduling}

In SP-RTS, partitions and channels are cyclically scheduled. The partition cyclic schedule is based on partition cycle, $\eta_{k}$, and partition capacity, $\alpha_{k}$. Similarly, a channel cyclic schedule with parameters, $\beta_{k}$ and $\mu_{k}$ implies that the channel can utilize $\beta_{k} \mu_{k}$ slots during a 
period of $\mu_{k}$ slot interval. While tasks and messages are scheduled according to their priority within the periodic servers, the cyclic schedule determines the response time of task execution and message transmission. In this subsection, we give a short description of the scheduling theory that can be used to schedule the cyclic partition and channel servers. A full discussion of the scheduling theory and the associated proof are given in our previous paper [10].

Note that, at the system level, the partition server $S_{k}$ is cyclically scheduled with a fixed partition cycle, $\eta_{k}$. For every partition cycle, the server can execute the task in partition $P_{k}$ during an interval of $\alpha_{k} \eta_{k}$ where $\alpha_{k} \leq 1$. For the remaining interval of $\left(1-\alpha_{k}\right) \eta_{k}$, the server is blocked. Suppose that there are $n$ tasks in partition server $S_{k}$ listed in priority order such that $\tau_{1}<\tau_{2}<\tau_{3}<\ldots<\tau_{n}$ where $\tau_{1}$ has the highest priority and $\tau_{n}$ the lowest. According to deadline monotonic algorithm, we assume that the highest priority is given to the task with shortest task deadline. In order to evaluate the schedulability of the partition server, $S_{k}$, we first consider that the task set is executed at a dedicated processor of capacity $\alpha_{k}$. Based on the necessary and sufficient condition of schedulability analysis $[12,13]$, task $\tau_{i}$ is schedulable if there exists a $t$ $\square H_{i}=\left\{C D_{i} \cup l T_{j} \mid j=1,2, \ldots, i-1 ; l=1,2, \ldots, \quad \bullet C D_{i} / T_{j} \bigcirc\right.$ Jsuch that:

$$
W_{i}\left(\alpha_{k}, t\right)=\sum_{j=1}^{i} \frac{C_{j}}{\alpha_{k}}\left\lceil\frac{t}{T_{j}}\right\rceil \leq t
$$

The expression $W_{i}\left(\alpha_{k}, t\right)$ indicates the worst cumulative execution time demand on the processor made by the tasks with a priority higher than or equal to $\tau_{i}$ during the interval $[0, t]$. We now define $B_{i}\left(\alpha_{k}\right)=\max _{t}{ }_{H i}\{t-$ $\left.W_{i}\left(\alpha_{k}, t\right)\right\}$ and $B_{0}\left(\alpha_{k}\right)=\min { }_{i=1,2, . . n} B_{i}\left(\alpha_{k}\right)$, where $n$ is the total number of tasks in the partition. Note that, when $\tau_{i}$ is schedulable, $B_{i}\left(\alpha_{k}\right)$ represent the total period in the interval $[0, t]$ that the processor is not running any tasks with a priority higher than or equal to that of $\tau_{i}$ in the partition server. $B_{i}\left(\alpha_{k}\right)$ is equivalent to the level- $i$ inactivity period in the interval $[0, t][11]$.

By comparing the task executions at server $S_{k}$ and at a dedicated processor of capacity $\alpha_{k}$, we can obtain the following theorem [10].

Theorem 1. The partition server $S_{k}$ is schedulable if $S_{k}$ is schedulable at a dedicated processor of capacity $\alpha_{k}$, and $\eta_{k} \leq B_{0}\left(\alpha_{k}\right) /\left(1-\alpha_{k}\right)$

Note that $B_{0}\left(\alpha_{k}\right)$ is a non-decreasing function of $\alpha_{k}$. There is a minimum $\alpha_{k}$ such that $B_{0}\left(\alpha_{k}\right)$ equals to zero, i.e., a zero inactive period for at least one task in the partition. The minimum $\alpha_{k}$ indicates the minimum processor capacity needed to schedule the partition. Thus, partition scheduling can fail if the sum of the minimum $\alpha_{k}$, for all partitions in a processor, is larger than 1 .

With Theorem 1, we can depict the plot of maximum partition cycle vs. the assigned capacity $\alpha_{k}$. To illustrate the result, we consider an example in Table 1 in which four application partitions are allocated in a processor. Each partition consists of several periodic tasks and the corresponding parameters of $\left(C_{i}, T_{i}\right)$ are listed in the Table. Tasks are set to have deadlines equal to their periods and are scheduled within each partition according to a rate-monotonic algorithm. The processor utilization demanded by the 4 partitions, $\rho_{k}$, are $0.25,0.15,0.27$, and 0.03 , respectively.

\section{Table 1. Task parameters for the example} partitions

\begin{tabular}{|c|c|c|}
\hline & $\begin{array}{c}\text { Partition 1 } \\
\text { (utilization=0.25) }\end{array}$ & $\begin{array}{c}\text { Partition 2 } \\
\text { (utilization=0.15) }\end{array}$ \\
\hline tasks & $(4,100)$ & $(2,50)$ \\
$\left(C_{i}, T_{i}\right)$ & $(9,120)$ & $(1,70)$ \\
& $(7,150)$ & $(8,110)$ \\
& $(15,250)$ & $(4,150)$ \\
& $(10,320)$ & \\
\hline & Partition 3 & Partition 4 \\
& (utilization=0.27) & $(1,80)$ \\
\hline tasks & $(7,80)$ & $(2,120)$ \\
$\left(C_{i}, T_{i}\right)$ & $(9,100)$ & \\
& $(16,170)$ & \\
\hline
\end{tabular}

In Figure 6, the curves $\eta_{k}=B_{0}\left(\alpha_{k}\right) /\left(1-\alpha_{k}\right)$ are plotted for the example 4 partitions. If the points below the curves are chosen to set up cyclic scheduling parameters for each partition, the tasks in the partition are guaranteed to meet their deadlines.

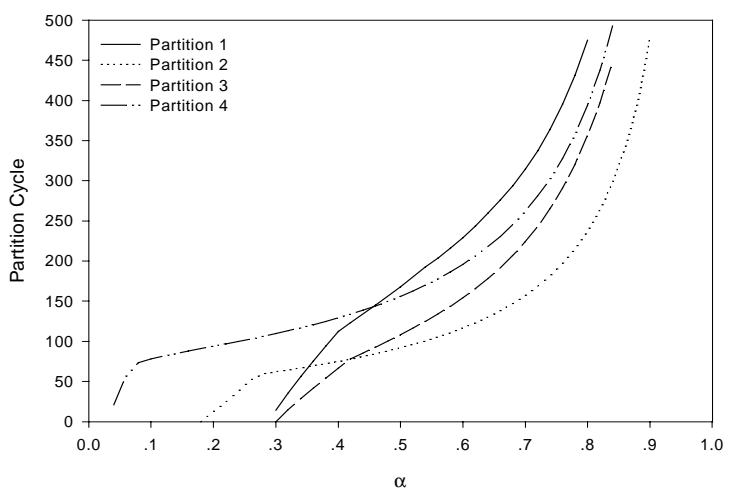

Figure 6. Partition Cycles vs. Processor Capacities for the Example Partitions

For instance, the curve for partition 2 indicates that, if the partition receives $28 \%$ of processor capacity, then its 
tasks are schedulable as long as its partition cycle is less than or equal to 59 time units. Note that the maximum partition cycles increase as we assign more capacity to each partition. This increase is governed by the accumulation of inactivity period when $\alpha_{k}$ is small. Then, the growth follows by a factor of $1 /\left(1-\alpha_{k}\right)$ for a larger $\alpha_{k}$. The curves in Figure 6 show that there are sharp rises of the maximum partition cycle when we increase $\alpha_{k}$ just beyond the minimum required capacities. The rises indicate that a small amount of extra capacity can enlarge the inactive period of a partition server significantly.

According to the design objectives, there are several methods we can use to choose a set of $\left(\alpha_{k}, \eta_{k}\right)$ for all partition servers. For instance, we can calculate the minimum $\alpha_{k}$ first. If the sum of the minimum $\alpha_{k}$, for all partition server $S_{k}$, and the reserved portion of processor capacity, is less than $100 \%$, the extra capacity can be allocated to all partitions proportionally to their minimum $\alpha_{k}$. Then, $\eta_{k}$ can be calculated based on Theorem 1 . The other approach is to search for the saddle point in the $B_{0}\left(\alpha_{k}\right) /\left(1-\alpha_{k}\right)$ curve where the initial rise just begins to slow down. The pair $\left(\alpha_{k}, \eta_{k}\right)$ at the saddle point is used as the initial capacity allocation and partition cycle. Further increase or reduction can be done proportionally if the total capacity allocated is less than or larger than 1.

We can use the same scheduling method of the partition scheduling for channel scheduling. A channel server, $G_{k}$, transmits its messages according to a fixed priority preemptive scheduling method. It provides a bandwidth of $\beta_{k} \mu_{k}$ slots to the messages in the channel during every channel cycle, $\mu_{k}$, where $\beta_{k} \leq 1$. For the remaining slots of $\left(1-\beta_{k}\right) \mu_{k}$, the channel server is blocked. Since each channel server follows the identical two-level hierarchical scheduling as partition servers, Theorem 1 can directly applied to obtain the pair of parameters $\left(\beta_{k}\right.$, $\left.\mu_{k}\right)$. However, there are several differences. First, only integer number of slots can be assigned to a channel server. Thus, we can use either $\left\lceil\beta_{k} \mu_{k}\right\rceil$ slots or restrict $\beta_{k} \mu_{k}$ to be integer. The second difference is that the message arrivals are not always periodic due to possible release jitters. Release jitters can be included in the schedulability test if they are bounded by some maximum value [15]. The release jitter can also be eliminated if the communication controller incorporates a timed message service that becomes active immediately after the computation deadline is expired. The last difference is the assignment of messages into a channel. According to the principle of partitioning, tasks from different partitions cannot share the same channel for message transmission. For the tasks in a partition, we can group a subset of tasks and let them share a channel server. The grouping can be done based on the semantics of the messages or other engineering constraints. Also, the multiplexing of messages in a shared channel may lead to a saving of bandwidth reservation. We should address this issue in the following subsection.

\subsection{Channel Combining}

For a channel server that transmits a periodic message with a deadline $M D_{i}$ and a message size $M_{i}$, we must allocate a minimum bandwidth of $M_{i} / M D_{i}$. Since there is a limitation in the total bus bandwidth, we may not always assign one channel server to each message. However, we may be able to combine some messages and let them share a common channel server. This can lead to a bandwidth reduction since the reserved bandwidth can be better utilized by the messages of different deadlines. For example, given two messages 1 and 2 with parameters $\left(M_{1}, M D_{1}, T_{1}\right)$ and $\left(M_{2}, M D_{2}, T_{2}\right)$, respectively, the minimum bandwidth requirements, in terms of slots per time unit, for separate channels of messages 1 and 2 , and for the combined channel, can be computed as following:

$$
\begin{aligned}
& C B_{1}=M_{1} / M D_{1}, \quad C B_{2}=M_{2} / M D_{2}, \\
& C B_{12}=\max \left\{M_{1} / M D_{1},\left(M_{2}+M_{1}^{*}\right. \text { erMD }\right. \\
& \left.\left.T_{1} \& \text { \& }\right) / M D_{2}\right\}
\end{aligned}
$$

We assume that message 1 has a higher priority than message 2 in the above computation. The cost of message preemption is ignored which can be at most one slot per preemption since we assume that slots are the basic transmission units in the communication bus. Notice that $C B_{12}$ is not always less that $C B_{1}+C B_{2}$. However, if message 1 has a much shorter deadline comparing with its period and message 2 has a longer deadline than message 1 's period, then the bandwidth reduction $C B_{1}+C B_{2}-C B_{12}$ becomes substantial. While we reserve a proper amount of bandwidth for an urgent message, the channel is only partially utilized if the message arrives infrequently. This provides a good chance to accommodate additional messages in the same channel and results in a reduction in the required bandwidth.

The above equation also implies that the maximum bandwidth reduction can be obtained by combining the message with a long deadline and the message with a short deadline where the period of the latter should be greater than message deadline of the former. With this observation, we devise a heuristic channel-combining algorithm which is shown in Figure 7. The computation of the minimum bandwidth requirement of a channel consisting of messages $1,2, \ldots, k-1$, and $k$, is:

$$
C B_{12 \ldots k}=\max _{j=1, k}\left\{\left(\left(\sum_{i=1}^{j-1} M_{i} *\left\lceil\frac{M D_{j}}{T_{i}}\right\rceil+M_{j}\right) / M D_{j}\right)\right\}
$$

where we assume that message $j$ has a higher priority then message $j+1$. Note that the real bandwidth allocation must be determined according to the choice of channel cycle as 
described in Theorem 1. However, in order to calculate channel cycle and capacity, the messages in each channel must be known. The channel-combining algorithm outlined in Figure 7 is developed to allocate messages to channels for each partition and to reduce the minimum bandwidth requirement to a specific threshold. If the combined channels cannot be scheduled, we can further decrease the target threshold until no additional combining can be done.

Initialization (Channel combining is allowed to the tasks in the same partition)

Assign one channel server $G_{k}$ to the message of each task

Iterate the following steps until the sum of total $C B_{k}$ is less than the target threshold

determine all pair of combinable channel server $G_{k}$ and $G_{j}$ where the max. message deadline in $G_{k}$ is larger than the min. task period in $G_{j}$

For every pair of combinable channel servers $G_{k}$ and $G_{j}\{$ calculate the bandwidth reduction $C B_{k}+C B_{j}-C B_{k j}$ \}

Combine $G_{j}$ with the server $G_{k}$ that results in the maximum reduction

Figure 7. A heuristic channel combining algorithm

\subsection{Cyclic Scheduling for Partition and Channel Servers}

Let a feasible set of partition capacities and cycles be $\left(\alpha_{1}, \eta_{1}\right),\left(\alpha_{2}, \eta_{2}\right), \ldots,\left(\alpha_{n}, \eta_{n}\right)$ and the set be sorted in the non-decreasing order of $\eta_{k}$. The set cannot be directly used in a cyclic schedule that guarantees the distance constraint of assigning $\alpha_{k}$ processor capacity for every $\eta_{k}$ period in a partition. To satisfy the distance constraint between any two consecutive invocations, we can adopt the pinwheel scheduling approach [7,9] and transfer $\left\{\eta_{k}\right\}$ into a harmonic set through a specialization operation. Note that, in [9], a fixed amount of processing time is allocated to each task and would not be reduced even if we invoke the task more frequently. This can lead to a lower utilization after the specialization operations. For our partition-scheduling problem, we allocate a certain percentage of processor capacity to each partition. When the set of partition cycles $\left\{\eta_{k}\right\}$ is transformed in to a harmonic set $\left\{h_{k}\right\}$, this percentage doesn't change. Thus, we can schedule any feasible sets of $\left(\alpha_{k}, \eta_{k}\right)$ as long as the total sum of $\alpha_{k}$ is less than 1 .

A simple solution for a harmonic set $\left\{h_{k}\right\}$ is to assign $h_{k}=\eta_{1}$ for all $k$. However, since it chooses a minimal invocation period for every partition, a substantial number of context switches between partitions could occur. A practical approach of avoiding excessive context switches is to use Han's $S_{X}$ specialization algorithm with a base 2 [9]. Given a base partition cycle $\eta$, the algorithm finds a $h_{i}$ for each $\eta_{i}$ that satisfies:

$$
h_{i}=\eta * 2^{j} \leq \eta_{i}<\eta * 2^{j+1}=2 * h_{i},
$$

To find the optimal base $\eta$ in the sense of processor utilization, we can test all candidates $\eta$ in the range of $\left(\eta_{1} / 2, \eta_{1}\right]$ and compute the total capacity $\sum_{k} \alpha_{k}$. To obtain the total capacity, the set of $\eta_{k}$ is transferred to the set of $h_{k}$ based on corresponding $\eta$ and then the least capacity requirement, $\alpha_{k}^{h}$, for partition cycle $h_{k}$ is obtained from Theorem 1. The optimal $\eta$ is selected in order to minimize the total capacity. In Figure 8, we show a fixed cyclic processor scheduling example that guarantees distance constraint for the set of partition capacities and cycles, $A(0.1,12), B(0.2,14), C(0.1,21)$, $D(0.2,25), E(0.1,48)$, and $F(0.3,50)$. We use the optimal base of 10 to convert the partition cycles to $10,10,20,20$, 40 , and 40 , respectively.

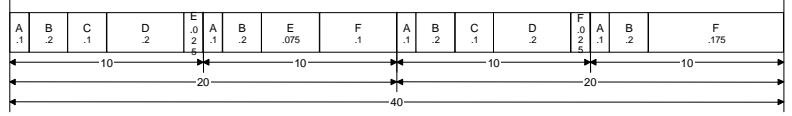

Figure 8. Example of processor cyclic scheduling

The basic method of cyclic scheduling for channel servers is same as that of partition server scheduling. The only difference is that we need to consider that channel bandwidth allocation must be done based on integer number of slots. Let the feasible bus bandwidth capacity allocation set be $\left(\beta_{1}, \mu_{1}\right),\left(\beta_{2}, \mu_{2}\right), \ldots,\left(\beta_{n}, \mu_{n}\right)$. Using the $S_{\mathrm{X}}$ specialization, the set $\left\{\mu_{k}\right\}$ will be transformed to a harmonic set $\left\{m_{k}\right\}$. Then, based on Theorem 1 and the reduced $m_{k}$, we can adjust the channel capacity $\beta_{k}$ to $\beta_{k}^{h}$ subject to $\sum_{i=1}^{n}\left\lceil\beta_{i}^{h} m_{k}\right\rceil \leq m_{k}$. There will be $\left\lceil\beta_{k}^{h} m_{k}\right\rceil$ slots allocated to the channel server $G_{k}$.

\section{Algorithm Evaluation}

In this section, we present the evaluation results of the proposed algorithms for SP-RTS. First, we show the percentage of schedulable task sets in terms of processor and bus utilization under the two-level scheduling, deadline decomposition and channel combining algorithms. Then, we show that the penalty of the 
$(\mathrm{N}, \mathrm{P}, \mathrm{T})=(4,3,5)$

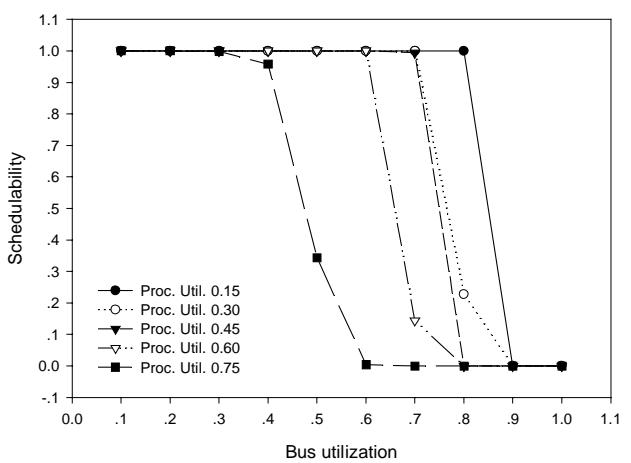

$(\mathrm{N}, \mathrm{P}, \mathrm{T})=(2,2,4)$

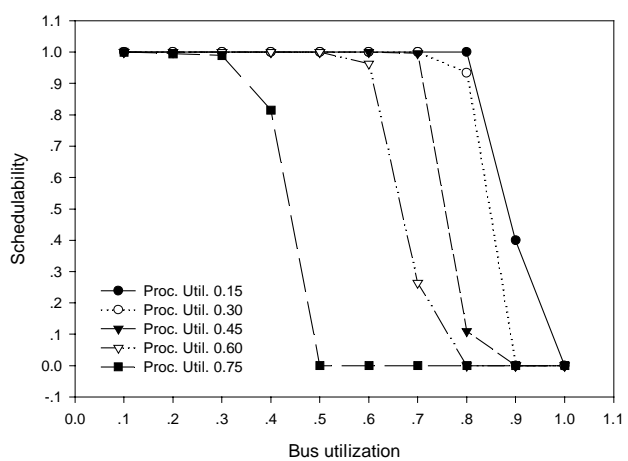

Figure 9 . Schedulability test for configurations $(4,3,5)$ and $(2,2,4)$

harmonic transformation even if channel server scheduling is negligibly small. Finally, the characteristic behavior of deadline decomposition is illustrated. The evaluations are done with random task and message sets that are generated with specific processor and bus utilization.

\subsection{Schedulability Test}

A schedulability test of the algorithm is obtained using the simulations of a system model that composes of four processors, three partitions per each processor and five tasks per each partition, i.e., a configuration of $(4,3,5)$. The simulations use random task sets that result in variable processor utilization of $15 \%, 30 \%, 45 \%, 60 \%$ and $75 \%$. The task periods are uniformly distributed between the minimum and maximum periods. The total processor utilization is randomly distributed to all tasks in each processor and is used to compute the task execution times. To create message sets, we vary the total bus utilization from $10 \%$ to $90 \%$. Message lengths are computed with a random distribution of the total bus utilization and task periods.

Using the scheduling procedure of Figure 4, we first assign task and message deadlines for each task. Then the partition capacity and cycle for each partition are computed and the cyclic schedule for each processor is constructed. To schedule message transmission, messages are combined into channels in order to reduce bandwidth requirement. After channel cycle and capacity are determined, a cyclic schedule is formed. For the priority schedules within partitions and channels, we adopt the deadline monotonic approach to order the task and message priorities. With all randomly created task sets, we report the percentage of schedulable task sets among all sets in Figure 9. The figure shows the algorithms are capable of finding proper deadline assignments and, then, determining feasible partition and channel cyclic schedules. For instance, consider the case of $60 \%$ processor and bus utilization. Even if the deadlines are less than task periods, almost $100 \%$ of task sets are schedulable. Figure 9 also reports the test results of the configuration $(2,2,4)$. The curves have the similar trends as that of the configuration of $(4,3,5)$.

\subsection{The Effects of Deadline Decomposition and Channel Combining Algorithm}

It is worthy to look into how the bus is utilized in the channel schedules resulted from the heuristic algorithms of deadline decomposition and channel combining. Consider the following measures:

1. Measurel is the bus utilization which equals to the sum of $\left(S T * M_{i}\right) / T_{i}$ for all tasks. No real-time constraint of message delivery is considered in this measure.

2. Measure 2 is the total bus capacity needed to transmit messages on time with no channel combining (i.e., each task has a dedicated channel). This capacity will be equal to the summation of $\left(S T * M_{i}\right) / M D_{i}$ for all tasks and can be computed after message deadlines are assigned.

3. Measure 3 is the minimum bus capacity needed to schedule channels. This measure is equal to the summation of minimum $\beta_{k}$ for all channels. Note that, according to Theorem 1 , the minimum $\beta_{k}$ for a channel is defined as the minimum 
capacity that results in a zero inactive period for at least one message in the channel. It can be determined after message deadlines are assigned and messages are combined into the channel.

4. Measure 4 is the total bus capacity selected according to Theorem 1. This measure can be formulated as the summation of $\beta_{k}$ for all channels.

5. Measure 5 is the final bus capacity allocated to all channels based on a harmonic set of channel cycles and the integer number of slots for each channel. The capacity is equal to the summation of $\left\lceil\beta_{k}{ }^{h} m_{k}\right\rceil / m_{k}$ for all channels.

We can expect an order of Measure2> Measure5> Measure4> Measure3> Measure1 among the measures. Measure 2 should be much higher than other measures as we allocate bandwidth for each message independently to ensure on schedule message delivery. With the message multiplexing within each channel, the on schedule message delivery can be achieved with a less amount of bandwidth. However, a bandwidth allocation following Measure3 cannot be practical since the channel cycles must be infinitely small. According to Theorem 1, Measure 4 contains additional capacity that is added to each channel to allow temporary blocking of message transmission during each channel cycle. Furthermore, in Measure5, an extra capacity is allocated as we make integer number of slots for each channel and construct a cyclic schedule with harmonic periods.

The simulation results of the above measures are shown in Figure 10. The results confirm our expectation of the order relationship. However, when we change the bus utilization from 0.1 to 0.8 , the curves are not monotonically increasing (except the curve of Measure1). This is the consequence of the deadline decomposition $(D D)$ algorithm. When channels don't have enough bandwidth to meet short message deadlines, the algorithm adjusts the factor $f_{k}$ and assigns longer deadlines for message transmission. As shown in Figure 5, the $D D$ algorithm uses an approach similar to binary search algorithm and makes a big increase to $f_{k}$ initially. This results in long deadlines and the reduced capacity allocations in Measure2-5. In fact, when the bus utilization is less than $30 \%$, the average number of iterations performed in the $D D$ algorithm is slightly larger than 1 , i.e., only the initial $f_{k}$ is used to allocate deadlines. When the bus utilization is raised to $40 \%$ to $70 \%$, the average number of iterations jumps to $1.6,1.98,2.0$, and 2.04 , respectively. It further increases to 11.09 when the bus utilization is set to $80 \%$.

Figure 10 also illustrates the magnitude of the measures and the differences among them. The gap between Measure 3 and Measure 2 is very visible. This difference is the product of channel combining algorithm.

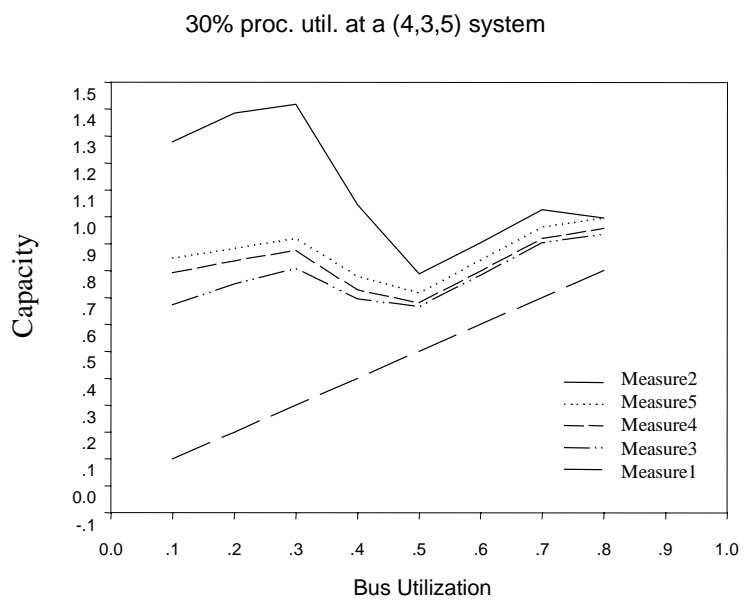

Figure 10. Measures for Bus Utilization and Capacities

In order to meet a tight message deadline, we have to reserve a large amount of bandwidth. With channel combining, messages of different deadlines share the allocated slots. As long as the message with a shorter deadline can preempt the on-going transmission, the slots in each channel can be fully utilized by multiplexing and prioritizing message transmissions. There is a moderate gap between Measure3 and Measure4. As indicated in Theorem 1, we search for a channel capacity and a channel cycle located in the knee of the curve $\eta_{k} \leq$ $B_{0}\left(\alpha_{k}\right) /\left(1-\alpha_{k}\right)$ after the initial sharp rise. This implies that a small increase of $\beta_{k}$ will be added to Measure 3 in order to obtain a reasonable size of channel cycle. Finally, the difference between Measure4 and Measure5 is not significant at all. It is caused by the process of converting $\eta_{k}$ to a harmonic cycle $m_{k}$, and by allocating an integer number of slots $\left\lceil\beta_{k}{ }^{h} m_{k}\right\rceil$ for each channel.

The other way of looking into the behavior of the deadline decomposition algorithm is to investigate the resultant decomposition of task deadline, $D_{i}$. In Figure 11, we showed the average ratio of message deadline to task deadline, under different processor and bus utilization. If the adjustment factor $f_{i}$ is constant, the ratio,

$$
\frac{M D_{i}}{D_{i}}=\left(\frac{S T * M_{i}}{C_{i}+S T * M_{i}}\right) f_{i},
$$

should follows a concave curve as we increase bus utilization (by increasing message length, $M_{i}$ ). For instance, when the processor utilization is $15 \%$, there are two segments of concave curves from bus utilization $10 \%$ to $70 \%$ and from $70 \%$ to $90 \%$. The segmentation indicates a jump in the adjustment factors resulted from the deadline decomposition algorithm. In Figure 11, the 
concavity and the segmentation can also be seen in other curves that represent the message deadline ratios of different processor utilization. When the processor utilization is high, $f_{i}$ may be modified gradually and partition scheduling may fail if we introduce a sharp increase to $f_{i}$. Thus, the concavity and the segmentation are not so obvious as the deadline ratio in an underutilized processor.

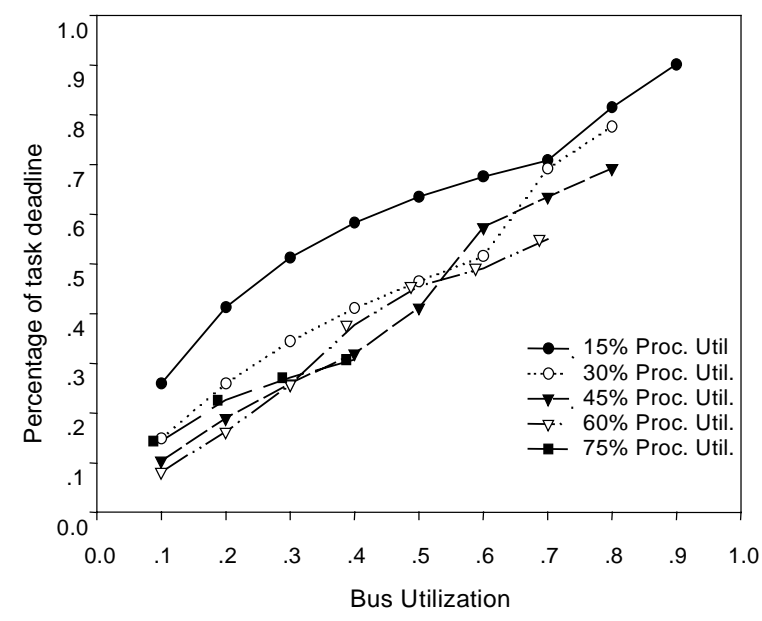

Figure 11. The ratio of message deadline to task deadline

\section{Conclusion}

In this paper, we present several algorithms in order to produce cyclic partition and channel schedules for the two-level hierarchical scheduling mechanism of IMAbased avionics systems. The system model of the IMA architecture supports spatial and temporal partitioning in all shared resources. Thus, applications can be easily integrated and maintained.

The main idea of our approach is to allocate a proper amount of capacity and to follow a distance constraint on partition and channel invocations. Thus, the tasks (messages) within a partition (channel) can have an inactive period longer than the blocking time of the partition (channel). Also we use a heuristic deadline decomposition technique to find feasible deadlines for both tasks and messages. To reduce bus bandwidth requirement for message transmission, we develop a heuristic channel-combining algorithm which leads to highly utilized channels by multiplexing messages of different deadlines and periods. The simulation analyses show promising results in terms of schedulability and system characteristics.

Based on the work in this paper, we have developed a scheduling tool for the IMA-based avionics systems. The tool includes additional features for practical implementations, such as time-tick based processor scheduling, non-zero context switch overhead, replication execution and transmission, incremental changes, etc. We are currently looking into different network infrastructures and communication scheduling algorithms that can be employed in the scalable IMA-based systems.

\section{References}

[1] "Design Guide for Integrated Modular Avionics," ARINC Report 651, Aeronautical Radio Inc., Annapolis, MD, Nov. 1991.

[2] "Avionics Application Software Standard Interface," ARINC Report 653, Aeronautical Radio Inc., Annapolis, MD, Jan. 1997.

[3] "Backplane Data Bus," ARINC Specification 659, Aeronautical Radio Inc., Annapolis, MD, Dec. 1993.

[4] N. Audsley, and A. Wellings, "Analyzing APEX applications," Proc. IEEE Real-Time Systems Symposium, Dec. 1996, pp. 39-44.

[5] N. Audsley, A. Burns, M. Richardson, and A. Wellings, "Hard real-time scheduling: the deadline-monotonic approach," Eighth IEEE Workshop on Real-time Operating Systems and Software, 1991, pp. 133-137.

[6] T. Carpenter, "Avionics Integration for CNS/ATM," Computer, Dec. 1998, pp. 124-126.

[7] M. Y. Chan and F. Y. L. Chin, "General schedulers for the pinwheel problem based on double-integer reduction," IEEE Trans. on Computers, vol. 41, June 1992, pp. 755-768.

[8] Z. Deng and J. W. S. Liu, "Scheduling real-time applications in an open environment," Proc. IEEE RealTime Systems Symposium, Dec. 1997, pp. 308-319.

[9] C.-C. Han, K.-J. Lin, and C.-J. Hou, "Distanceconstrained scheduling and its applications to real-time systems," IEEE Trans. on Computers, Vol. 45, No. 7, July, 1996, pp. 814--826.

[10] Y. H. Lee, D. Kim, M. Younis, and J. Zhou, "Partition scheduling in APEX runtime environment for embedded avionics software," Proc. of Real-Time Computing Systems and Applications, Oct. 1998, pp. 103-109.

[11] J. Lehoczky and S. Ramos-Thuel, "An optimal algorithm for scheduling soft-aperiodic tasks in fixed-priority preemptive systems," Proc. IEEE Real-Time Systems Symposium, Dec. 1992, pp. 110-123.

[12] J. Lehoczky, L. Sha, and Y.Ding, "The rate-monotonic scheduling algorithm: exact characteristics and average case behavior," Proc. IEEE Real-Time Systems Symposium, Dec. 1989, pp. 166-171.

[13] J. Lehoczky, "Fixed priority scheduling for periodic task sets with arbitrary deadlines," Proc. IEEE Real-time Systems Symposium, Dec. 1990, pp. 201-209.

[14] C. L. Liu and J. W. Layland, "Scheduling algorithms for multiprogramming in a hard real-time environment," JACM, vol. 20, No. 1, 1973, pp.46-61.

[15] K. W. Tindell, A. Burns, and A. J. Wellings, "An extendible approach for analyzing fixed priority hard real-time tasks," Real-Time Systems 6(2), 1994, pp. 133 151. 